\title{
Evaluation of Patients with High National Institutes of Health Stroke Scale as Thrombectomy Candidates Using the Kentucky Appalachian Stroke Registry
}

\author{
Parneet Grewal ${ }^{\mathrm{a}} \quad$ Michael R. Dobbs $^{f}$ Keith Pennypacker ${ }^{\mathrm{a}} \quad$ Richard J. Kryscio $^{\mathrm{e}}$ \\ Patrick Kitzman $^{f}$ Marc Wolfe ${ }^{\mathrm{f} g}$ Kelley Elkins ${ }^{g}$ Gregory J. Bix ${ }^{d}$ \\ Justin F. Fraser ${ }^{\mathrm{a}-\mathrm{c}}$ \\ a Department of Neurology, Rush University Medical Center, Chicago, IL, USA; b Department of Neurological Surgery, \\ College of Medicine/University of Kentucky, Lexington, KY, USA; ' Department of Radiology, College of Medicine/ \\ University of Kentucky, Lexington, KY, USA; ${ }^{d}$ Department of Neurosurgery, Tulane University, New Orleans, LA, USA; \\ e Department of Statistics, College of Arts and Sciences/University of Kentucky, Lexington, KY, USA; f Department of \\ Neurology, University of Texas Rio Grande, Rio Grande, TX, USA; ${ }^{9}$ Norton Healthcare/UK HealthCare Stroke Network, \\ Lexington, KY, USA
}

\author{
Keywords \\ Stroke · National Institutes of Health Stroke Scale $\cdot$ Rural \\ health - Thrombectomy
}

\begin{abstract}
Introduction: Mechanical thrombectomy has become standard of care for emergent large vessel occlusive stroke. Estimates of incidence for thrombectomy eligibility vary significantly. National Institutes of Health Stroke Scale (NIHSS) of 10 or greater is highly predictive of large vessel occlusion. Using our Kentucky Appalachian Stroke Registry (KApSR), we evaluated temporal trends in stroke admissions with NIHSS $\geq 10$ to determine patient characteristics among that group along with effects and needs in thrombectomy utilization. Methods: Using the KApSR database that captures patients throughout the Appalachian region in our stroke network, we evaluated patients admitted with ischemic stroke with NIHSS $\geq 10$. We recorded demographics, comorbidities, treatment (thrombectomy, decompressive craniectomy),
\end{abstract}

and county of origin. Change in NIHSS from admission to discharge was used as an indicator of inpatient outcome. $\boldsymbol{R} \boldsymbol{e}$ sults: Between 2010 and 2016, 1,510 patients were admitted with NIHSS $\geq 10.87 .2 \%$ had high blood pressure, $69.6 \%$ had dyslipidemia, and $41.7 \%$ used tobacco. There were significant sex differences in the types of patients presenting with NIHSS $\geq 10$ with females being older on average and having more atrial fibrillation and obesity. There was an increase in thrombectomy utilization from 2010 to 2016 , but only $7.5 \%$ of the potentially eligible patients underwent the procedure. In comparison to the period 2010-2014, the 20152016 period had higher rates of obesity and tobacco abuse. Conclusion: Among patients with significant burden of ischemic stroke, the most common coexisting medical condition was high blood pressure. Patients who underwent thrombectomy had significantly better inpatient clinical improvement. These data support the need to maximize utilization of thrombectomy along with need to devote increased resources on modifiable stroke risk factors.

(c) 2019 S. Karger AG, Basel

\section{KARGER}

(c) 2019 S. Karger AG, Basel

E-Mail karger@karger.com

www.karger.com/ced
Justin F. Fraser, MD, FAANS, FAHA

Departments of Neurological Surgery, Neurology, Radiology, and Neuroscience Center for Advanced Translational Stroke Science, University of Kentucky 800 Rose Street, Room MS105B, Lexington, KY 40536 (USA)

E-Mail jfr235@uky.edu 


\section{Introduction}

Ischemic stroke accounts for approximately $87 \%$ of stroke [1], with emergent large vessel occlusions (ELVO) representing 28-46\% [2]. The presence of ELVO has been shown in various studies to increase odds of death by 4.5 fold and to cause a 3-fold reduction of good outcome in affected populations [2]. The goal of current therapeutic strategies is recanalization of the occlusion before irreversible damage has occurred. Intravenous tissue plasminogen activator (IV-tPA) given up to $4.5 \mathrm{~h}$ after onset of symptoms has been the only standard of care for acute ischemic strokes in the past [3], even though in cases of proximal vessel occlusion it only results in early reperfusion in $13-50 \%$ of cases [4]. More recently, endovascular mechanical thrombectomy (MT) has become a standard of care procedure with established benefits shown in multiple clinical trials [4-7]. More recently, with the use of perfusion-based imaging and automated software, this time window has been extended up to $24 \mathrm{~h}$ in a selected patient population with target mismatch $[8,9]$. Estimates of incidence for thrombectomy eligibility vary significantly across the nation. The National Institutes of Health Stroke Scale (NIHSS) is a validated tool to assess stroke severity and scores $\geq 10$ have been associated with positive predictive value of $97 \%$ in carotid and $96 \%$ in vertebro-basilar occlusions [10].

High stroke incidence and stroke risk factor burden in West Virginia and rural Kentucky have led to development of the Kentucky Appalachian Stroke Registry (KApSR) to collect demographic and clinical data in real time from stroke patients' electronic health records and to utilize this information for quality of care and research [11]. The aim of this study was to evaluate temporal trends in regional stroke admissions with NIHSS $\geq 10$ to determine epidemiologic effects and needs in thrombectomy utilization using the KApSR.

\section{Methods}

Using the previously described KApSR methodology [11], a snapshot database was created for cross-sectional analysis of patients over 18 years of age, admitted from January 1, 2010, to December 31, 2016, to the University Hospital with acute ischemic stroke or transient ischemic attack. These patients were studied retrospectively, and the patients with NIHSS $\geq 10$ recorded on admission were selected for further analysis. This was based on the hypothesis that the patients with NIHSS $\geq 10$ have a very high likelihood of having a proximal vessel occlusion and are potential thrombectomy candidates. Demographic characteristics were obtained and studied. We also analyzed whether these patients re- ceived intravenous thrombolytic, underwent thrombectomy, or underwent decompressive hemicraniectomy during their hospitalization. Change in NIHSS from admission to discharge was measured as an indicator of inpatient outcome [12]. The data were also analyzed for gender differences. Finally, we analyzed subgroups of epochs before (2010-2014) and after (2015-2016) AHA/ ASA guidelines recommended thrombectomy as a standard for ELVO [13].

Summary statistics and analyses were performed on PC - SAS version 9.1, and $p \leq 0.05$ was considered significant. Proportion was compared using chi-square statistics, and means were compared using 2 sample $t$ tests. The research protocol was approved by our Institutional Review Board, which waived informed consent for study subjects due to the retrospective nature of the study.

\section{Results}

\section{Population Characteristics and Risk Factors}

In total, 1,510 adult patients had NIHSS $\geq 10$ recorded at presentation between 2010 and 2016 and were selected for analysis. Table 1 provides the demographic, comorbidity, and treatment details for the cohort. The mean age of patients was $68.15 \pm 14.65$ with average NIHSS on admission of $17.58 \pm 6.24$. The most common comorbidity was hypertension (87.2\% of patients), followed by dyslipidemia (69.6\%), tobacco use (41.7\%), and coronary artery disease (CAD; $41.1 \%)$. Females were, on average, 5 years older and were significantly more likely to have atrial fibrillation (AF) and obesity. Conversely, males used tobacco more frequently and were more likely to have CAD. Figure 1 illustrates significant differences in patients admitted with NIHSS $\geq 10$ between the 2 time periods 2010-2014 and 2015-2016. Patients admitted in the later epoch had lower rates of CAD (44.1 vs. $34.9 \%$; $p<0.001)$ and dyslipidemia (71.7 vs. $65.3 \%$; $p=0.01)$. However, comparing time epochs, patients in the later cohort were younger (68.8 vs. 66.9 years; $p=$ 0.02 ), had higher rates of tobacco use (38.5 vs. $48.0 \%$; $p<$ 0.001 ), and had higher rates of obesity ( 8.9 vs. $18.7 \% ; p<$ $0.001)$.

\section{Treatments Administered}

Among 1,510 patients, 308 patients (20.4\%) with NIHSS $\geq 10$ on admission underwent treatment with intravenous thrombolysis between 2010 and 2016. Thrombectomy was performed in 113 patients (7.5\%) only with no significant difference between sexes in terms of utilization. Annual utilization of thrombectomy was plotted and showed an increase over time from $0.8 \%$ in 2010 to $9.4 \%$ in 2016 (Fig. 2). On comparison between the 2010 2014 and 2015-2016 periods, it was found that patients in 
Fig. 1. Comparison between 2010-2014 and 2015-2016 with significant differences.

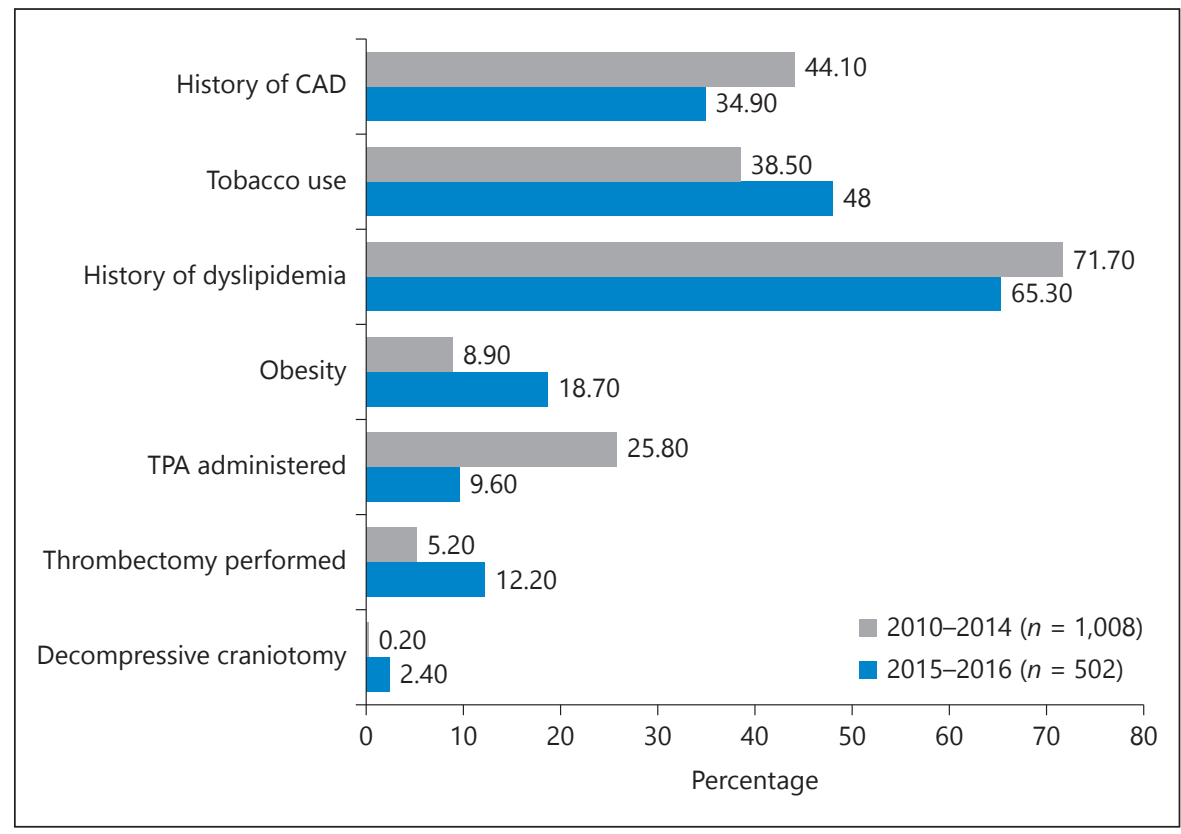

Table 1. Population demographics with comparisons between the male and female patient population

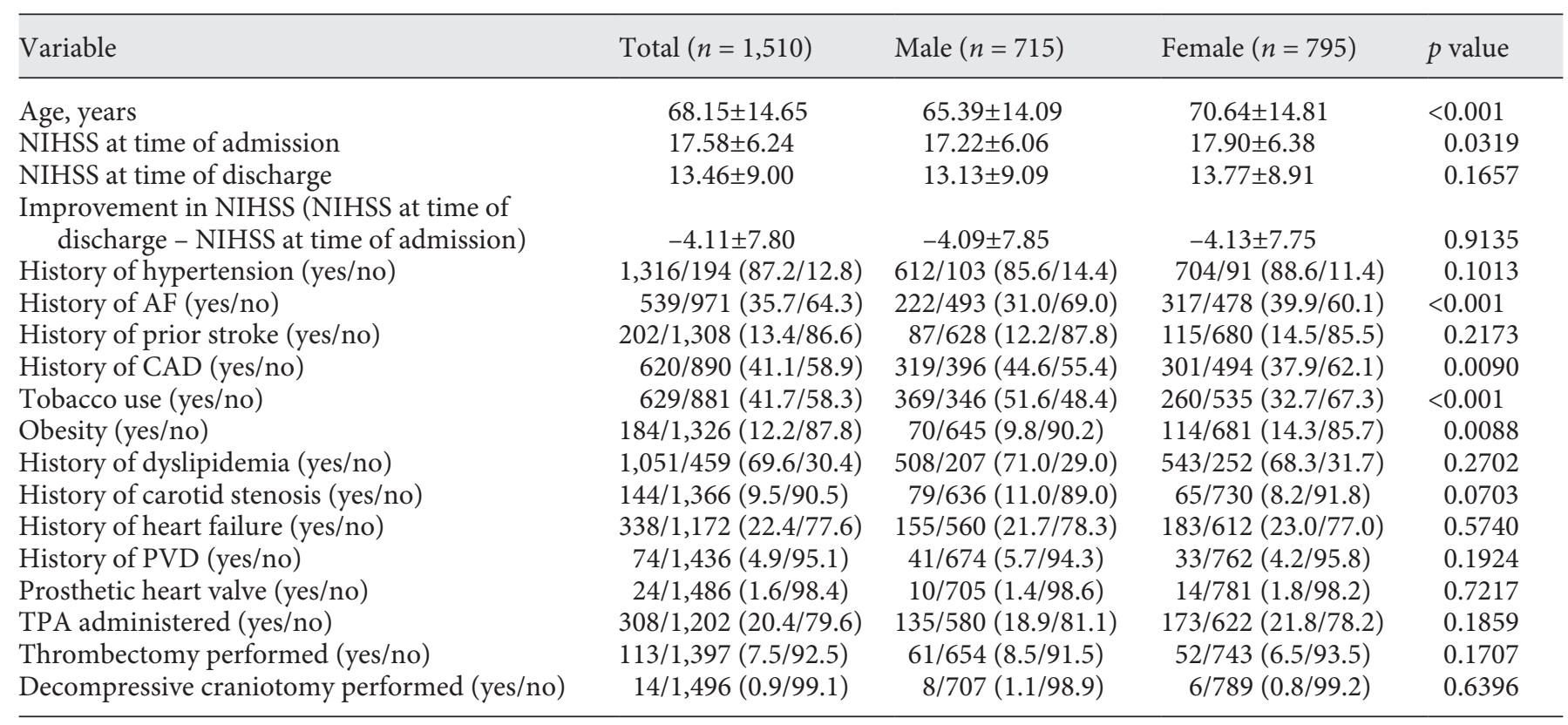

NIHSS, National Institutes of Health Stoke Scale; AF, atrial fibrillation; CAD, coronary artery disease; TPA, tissue plasminogen activator; PVD, peripheral vascular disease.

the later epoch were almost twice as likely to get a thrombectomy (12.2 vs. $5.2 \%$; $p<0.001)$ along with increase in decompressive craniectomy ( 2.4 vs. $0.2 \% ; p<0.001)$. The percentage use of tPA declined in the later time period (from 25.8 to $9.6 \% ; p<0.001$ ).

Patients with High NIHSS as Thrombectomy Candidates

\section{Outcome Measures}

Comparison of the mean NIHSS score change from admission to discharge between those who underwent MT and those who did not show a significant difference $(-5.78 \pm 9.09$ vs. $-3.98 \pm 7.67 ; p=0.04)$ with the biggest 
Fig. 2. The figure shows number of thrombectomies performed per year since 2010 . The annual rate of MT significantly increased from 2010 to 2016 with maximum increase from 2014 to 2015.

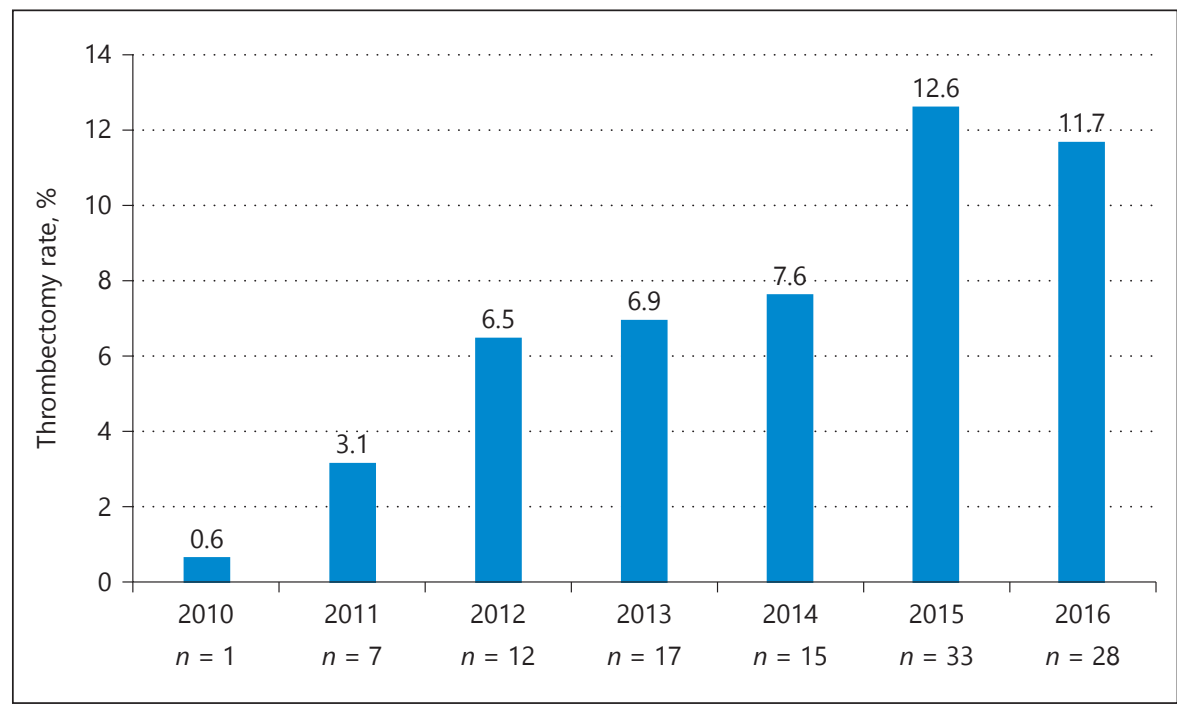

Table 2. Inpatient NIHSS outcomes and length of stay in patients that underwent thrombectomy as compared to the patients that did not undergo the procedure

\begin{tabular}{llll}
\hline & $\begin{array}{l}\text { Thrombectomy } \\
\text { group }\end{array}$ & $\begin{array}{l}\text { No thrombectomy } \\
\text { group }\end{array}$ & $p$ value \\
\hline NIHSS score change $(n=1,510)$ & $-5.78 \pm 9.09$ & $-3.98 \pm 7.67$ & 0.04 \\
NIHSS score change in year 2010-2014 $(n=1,008)$ & $-5.00 \pm 9.17$ & $-3.97 \pm 7.74$ & 0.43 \\
NIHSS score change in year 2015-2016 $(n=502)$ & $-6.44 \pm 9.04$ & $-3.99 \pm 7.53$ & 0.047 \\
LOS $(n=1,510)$ & $14.75 \pm 36.42$ & $10.14 \pm 14.30$ & 0.18 \\
LOS in year 2010-2014 $(n=1,008)$ & $13.52 \pm 24.36$ & $10.10 \pm 12.41$ & 0.32 \\
LOS in year 2015-2016 $(n=502)$ & $15.80 \pm 44.38$ & $10.23 \pm 17.76$ & 0.34 \\
\hline
\end{tabular}

NIHSS, National Institutes of Health Stoke Scale; MTY, mechanical thrombectomy yes; MTN, mechanical thrombectomy no; LOS, length of stay.

difference noted in year 2015-2016 (NIHSS decrease of 6.4 in thrombectomy patients vs. 4.0 in nonthrombectomy patients; $p=0.05$ ). The "No Thrombectomy" group had a slightly but not significantly shorter length of stay. Analysis of LOS and NIHSS change by sex showed a significant difference in mean LOS, with males having longer LOS (14.55 vs. $10.2 ; p=0.0054$; Table 2).

\section{Discussion}

We analyzed the temporal trends in thrombectomy at a regional stroke center serving a primarily rural US population. Our Comprehensive Stroke Center receives thrombectomy patients from central Kentucky, northern Tennessee, and western West Virginia. As such, many of our patients originate from the Appalachian region, where stroke represents a major health disparity. Rural cohorts in regions such as Appalachia have been shown to have higher rates of stroke, even after accounting for comorbidities and other risk factors [14]. Furthermore, unlike large urban environments, central Kentucky has only 2 Comprehensive Stroke Centers (with only 4 in the entire state). Treatment of severe stroke, therefore, tends to be centralized in the 2 largest cities (Louisville for western Kentucky and Lexington for central and eastern Kentucky). In that setting, we aimed to understand the patient population presenting with high NIH stroke scales, which represents patients most likely to have an Emergent Large Vessel Occlusion. Despite the growing evidence supporting MT, only $7.5 \%$ of the patients that presented to our hospital with NIHSS $\geq 10$ underwent thrombectomy, highlighting the need for additional edu- 
cation and collaboration with referring hospitals and emergency medical service providers; this is particularly important, as the window for thrombectomy has been expanded to $24 \mathrm{~h} \mathrm{[9]}$.

As shown by our data, the burden of comorbidities suffered by patients in the rural Kentucky and broader Appalachian region with acute ischemic stroke is significant. Even though AF is one of the main causes of vessel occlusion, the incidence of $\mathrm{AF}$ in our patient population was low as compared to hypertension, dyslipidemia, and tobacco abuse. This stresses the need to devote more resources toward management of these chronic medical conditions. Furthermore, we showed significant differences in the rates and types of comorbidities among men and women in our region. While men had higher rates of $\mathrm{CAD}$ and tobacco use, women had higher rates of $\mathrm{AF}$ and obesity. These findings are consistent with some other published studies evaluating sex differences in stroke [15-17]. Tobacco use, CAD, AF, and obesity are all linked to increased inflammation, which is a major factor in stroke susceptibility [18-21]. Interestingly, the behaviors or pathologies associated with inflammation linked to stroke differ between the sexes. These represent important sex-specific modifiable risk factors, which should be targets for public policy intervention regarding stroke prevention.

In addition to sex-based differences in high-NIHSS stroke incidence, we also noted changing characteristics among the patients in 2 sequential epochs. While overall rates of CAD and dyslipidemia decreased from 20112014 to 2015-2016 among the cohort, rates of obesity and tobacco usage increased. Elevated body mass index in particular has been linked to increased stroke mortality in younger patients [22]. As stated above, obesity and tobacco use are both associated with inflammation [20]. Since inflammation is prothrombotic [23], obesity, and tobacco use among younger people could be synergistic in decreasing the age of onset for stroke. This reflects the growing and important role that obesity has in the rural population as a modifiable stroke risk factor. Most importantly, the mean age of patients presenting with NIHSS of 10 or more was lower in the later cohort. This is concerning, since it reflects an epidemiologic shift toward younger age severe stroke incidence in our region.

There are several limitations to our study. First, the retrospective nature limits the ability to perform wellcontrolled and designed comparisons. Second, our analysis reflects patients presenting with NIHSS $\geq 10$ specifically rather than confirmed ELVO. While the 2 have been shown to be closely associated, they are not the same. This limitation of data extrapolation through this methodology is that individual patients cannot be evaluated in terms of protected health information. Therefore, there are variables we could not incorporate. For example, the rate of tPA usage in this population went down from the earlier to later epoch. However, this is likely explained by expanded indications for transfer of ELVO patients for thrombectomy. If more patients arrived at our institution in the $>4.5 \mathrm{~h}$ window, then the number of tPA-eligible patients with high NIHSS would be lower as a result. Finally, while our goal was to provide a region-specific analysis of patients with severe ischemic stroke, extrapolation of our results to another geographic region may not be valid.

\section{Conclusions}

Estimates of thrombectomy eligibility and utilization differ across geographic regions. In Appalachia, evaluation of the patients with severe NIH stroke scales suggests gross underutilization of MT. As the age of stroke patients in the region is decreasing, the need for additional resources to treat severe stroke as a community burden is intensifying. In creating prevention and treatment policies, we should consider notable sex differences in known risk factors, and use that information to design-specific strategies to reduce severe ischemic stroke incidence, and to rapidly treat it with thrombectomy when appropriate.

\section{Funding Source}

This publication was supported by the National Center for Research Resources and the National Center for Advancing Translational Sciences, National Institutes of Health, through Grant UL1TR001998. The content is solely the responsibility of the authors and does not necessarily represent the official views of the NIH.

\section{Statement of Ethics}

The research protocol was approved by our Institutional Review Board.

\section{Disclosure Statement}

The authors have no conflicts of interest to declare. 


\section{Author Contributions}

P.G.: study design, interpretation of data, manuscript writing, and approval of final manuscript. M.R.D. and J.F.F.: study design, interpretation of the data, and critical revision of the manuscript for intellectual content. K.P., P.K., M.W., and G.J.B.: critical revision of the manuscript for intellectual content and approval of final manuscript. R.J.K.: interpretation of the data, statistical analysis, and critical revision of the manuscript for intellectual content. K.E.: acquisition of data and approval of final manuscript.

\section{References}

1 Ovbiagele B, Nguyen-Huynh MN. Stroke epidemiology: advancing our understanding of disease mechanism and therapy. Neurotherapeutics. $2011 \mathrm{Jul} ; 8(3): 319-29$.

2 Smith WS, Lev MH, English JD, Camargo EC, Chou M, Johnston SC, et al. Significance of large vessel intracranial occlusion causing acute ischemic stroke and TIA. Stroke. 2009 Dec;40(12):3834-40.

3 Hacke W, Kaste M, Bluhmki E, Brozman M, Dávalos A, Guidetti D, et al.; ECASS Investigators. Thrombolysis with alteplase 3 to 4.5 hours after acute ischemic stroke. N Engl J Med. 2008 Sep;359(13):1317-29.

4 Saver JL, Goyal M, Bonafe A, Diener HC, Levy EI, Pereira VM, et al.; SWIFT PRIME Investigators. Stent-retriever thrombectomy after intravenous t-PA vs. t-PA alone in stroke. $\mathrm{N}$ Engl J Med. 2015 Jun;372(24):2285-95.

5 Berkhemer OA, Fransen PS, Beumer D, van den Berg LA, Lingsma HF, Yoo AJ, et al.; MR CLEAN Investigators. A randomized trial of intraarterial treatment for acute ischemic stroke. N Engl J Med. 2015 Jan;372(1):1120.

6 Campbell BC, Mitchell PJ, Kleinig TJ, Dewey HM, Churilov L, Yassi N, et al.; EXTEND-IA Investigators. Endovascular therapy for ischemic stroke with perfusion-imaging selection. N Engl J Med. 2015 Mar;372(11):1009-18.

7 Goyal M, Demchuk AM, Menon BK, Eesa M, Rempel JL, Thornton J, et al.; ESCAPE Trial Investigators. Randomized assessment of rapid endovascular treatment of ischemic stroke. N Engl J Med. 2015 Mar;372(11): 1019-30.

8 Albers GW, Marks MP, Kemp S, Christensen S, Tsai JP, Ortega-Gutierrez S, et al.; DEFUSE
3 Investigators. Thrombectomy for Stroke at 6 to 16 Hours with Selection by Perfusion Imaging. N Engl J Med. 2018 Feb;378(8):70818.

9 Nogueira RG, Jadhav AP, Haussen DC, Bonafe A, Budzik RF, Bhuva P, et al.; DAWN Trial Investigators. Thrombectomy 6 to 24 Hours after Stroke with a Mismatch between Deficit and Infarct. N Engl J Med. 2018 Jan; 378(1):11-21.

10 Fischer U, Arnold M, Nedeltchev K, Brekenfeld C, Ballinari P, Remonda L, et al. NIHSS score and arteriographic findings in acute ischemic stroke. Stroke. 2005 Oct;36(10):2121-5.

11 Kitzman P, Wolfe M, Elkins K, Fraser JF, Grupke SL, Dobbs MR. The Kentucky Appalachian Stroke Registry (KApSR). J Stroke Cerebrovasc Dis. 2018 Apr;27(4):900-907.

12 Dobbs MR, Krishnamohan P, Jicha G, Cohen AP. Innovation in Stroke Care Quality: NIH Stroke Scale Change and Shewhart Charts. Qual Manag Health Care. 2015 Jul-Sep;24(3): 135-9.

13 Powers WJ, Derdeyn CP, Biller J, Coffey CS, Hoh BL, Jauch EC, et al.; American Heart Association Stroke Council. 2015 American Heart Association/American Stroke Association Focused Update of the 2013 Guidelines for the Early Management of Patients With Acute Ischemic Stroke Regarding Endovascular Treatment: A Guideline for Healthcare Professionals From the American Heart Association/American Stroke Association. Stroke. 2015 Oct;46(10):3020-35.

14 Kapral MK, Austin PC, Jeyakumar G, Hall R, Chu A, Khan AM, et al. Rural-Urban Differences in Stroke Risk Factors, Incidence, and Mortality in People With and Without Prior
Stroke. Circ Cardiovasc Qual Outcomes. 2019 Feb;12(2):e004973.

15 Spychala MS, Honarpisheh P, McCullough LD. Sex differences in neuroinflammation and neuroprotection in ischemic stroke. Neurosci Res. 2017 Jan;95(1-2):462-71.

16 Emdin CA, Wong CX, Hsiao AJ, Altman DG, Peters SA, Woodward M, et al. Atrial fibrillation as risk factor for cardiovascular disease and death in women compared with men: systematic review and meta-analysis of cohort studies. BMJ. 2016 Jan;532:h7013.

17 Ball J, Carrington MJ, Wood KA, Stewart S; SAFETY Investigators.. Women versus men with chronic atrial fibrillation: insights from the Standard versus Atrial Fibrillation spEcific managemenT studY (SAFETY). PLoS One. 2013 May;8(5):e65795.

18 Smoking and Inflammation. Smoking and Inflammation. PLoS Med. 2005;2(6):e198.

19 Hu YF, Chen YJ, Lin YJ, Chen SA. Inflammation and the pathogenesis of atrial fibrillation. Nat Rev Cardiol. 2015 Apr;12(4):230-43.

20 Ellulu MS, Patimah I, Khaza'ai H, Rahmat A, Abed Y. Obesity and inflammation: the linking mechanism and the complications. Arch Med Sci. 2017 Jun;13(4):851-63.

21 McColl BW, Allan SM, Rothwell NJ. Systemic infection, inflammation and acute ischemic stroke. Neuroscience. 2009 Feb;158(3):104961.

22 Darke S, Duflou J, Kaye S, Farrell M, Lappin J. Body mass index and fatal stroke in young adults: A national study. J Forensic Leg Med. 2019 Apr;63:1-6.

23 Libby P, Simon DI. Inflammation and thrombosis: the clot thickens. Circulation. $2001 \mathrm{Apr}$; 103(13):1718-20. 